\title{
Effects of wave shape on sheet flow sediment transport
}

\author{
Tian-Jian $\mathrm{Hsu}^{1}$ \\ Center for Applied Coastal Research, University of Delaware, Newark, Delaware, USA
}

Daniel M. Hanes ${ }^{2}$

Western Coastal and Marine Geology, U.S. Geological Survey Pacific Science Center, Santa Cruz, California, USA

Received 30 July 2003; revised 19 March 2004; accepted 1 April 2004; published 25 May 2004.

[1] A two-phase model is implemented to study the effects of wave shape on the transport of coarse-grained sediment in the sheet flow regime. The model is based on balance equations for the average mass, momentum, and fluctuation energy for both the fluid and sediment phases. Model simulations indicate that the responses of the sheet flow, such as the velocity profiles, the instantaneous bed shear stress, the sediment flux, and the total amount of the mobilized sediment, cannot be fully parameterized by quasi-steady free-stream velocity and may be correlated with the magnitude of local horizontal pressure gradient (or free-stream acceleration). A net sediment flux in the direction of wave advance is obtained for both skewed and saw-tooth wave shapes typical of shoaled and breaking waves. The model further suggests that at critical values of the horizontal pressure gradient, there is a failure event within the bed that mobilizes more sediment into the mobile sheet and enhances the sediment flux. Preliminary attempts to parameterize the total bed shear stress and the total sediment flux appear promising. INDEX TERMS: 4558 Oceanography: Physical: Sediment transport; 4546 Oceanography: Physical: Nearshore processes; 3022 Marine Geology and Geophysics: Marine sediments_processes and transport; 4568 Oceanography: Physical: Turbulence, diffusion, and mixing processes; KEYWORDS: sediment transport, bed shear stress, sheet flow

Citation: Hsu, T.-J., and D. M. Hanes (2004), Effects of wave shape on sheet flow sediment transport, J. Geophys. Res., 109, C05025, doi:10.1029/2003JC002075.

\section{Introduction}

[2] One of the most important but relatively unknown aspects of wave-induced sediment transport and cross-shore profile evolution is the mechanism through which waves transport sediment onshore to counteract the effects of gravity. A key parameter for cross-shore sediment transport under breaking and near-breaking waves is the shape of the near-bed wave orbital velocity. It is generally believed that a skewed velocity field can cause a net cross-shore transport of sediment without a net transport of water, though the detailed mechanics of this process are not well understood. From a parameterization point of view, there is significant experimental evidence that flow acceleration, which serves as a proxy for horizontal pressure gradient in coastal bottom boundary layer, has an effect on sediment transport. This evidence derives from U-tube experiments [e.g., King, 1990] and field measurements in the surf zone [e.g., Hanes and Huntley, 1986; Gallagher et al., 1998] and in the swash [e.g., Butt and Russell, 1999; Puleo et al., 2003]. Motivated in part by King's [1990] measured sediment transport rates

\footnotetext{
${ }^{1}$ Now at Applied Ocean Physics and Engineering Department, Woods Hole Oceanographic Institution, Woods Hole, Massachusetts, USA.

${ }^{2}$ Also at Department of Civil and Coastal Engineering, University of Florida, Gainesville, Florida, USA.

Copyright 2004 by the American Geophysical Union. 0148-0227/04/2003JC002075\$09.00
}

under saw-tooth shaped waves, Nielsen [1992] proposed an empirical formula that estimates the Shields parameter based on both flow velocity and acceleration. More recently, Nielsen [2002] and Nielsen and Callaghan [2003] implemented a modified version of the formula for the Shields parameter proposed by Nielsen [1992] and applied it to predict sediment transport rate measurements in the swash zone [Masselink and Hughes, 1998] and to measurements of sheet flow in a large-scale wave flume under non-breaking waves [Dohmen-Janssen and Hanes, 2002]. Although the formula proposed by Nielsen and his colleagues has achieved a certain degree of success in predicting the sediment transport rate under unsteady conditions, a more complete theoretical background for the unsteady effect on bed shear stress and corresponding sediment transport is warranted.

[3] Numerical simulations of bedload sediment transport have also indicated that the sediment flux is influenced by flow acceleration. Drake and Calantoni [2001] proposed a parameterization of acceleration effects based upon discrete element simulations. Recently, Hoefel and Elgar [2003] use the acceleration skewness parameterization suggested by Drake and Calantoni [2001] in combination with the commonly used energetics-based total load formula of Bailard [1981]. They are able to predict the beach profile evolution, including both onshore and offshore sandbar migrations, over a 45-day period during the Duck94 experiments [Elgar et al., 2001]. 
[4] On the contrary, recent laboratory measurements of sediment transport under sheet flow condition in U-tubes or under nonbreaking waves suggest that most of the transport occurs in the concentrated region near the bed where the time evolution of sediment concentration responses directly to the free-stream velocity [e.g., Ribberink, 1998; DohmenJanssen and Hanes, 2002]. In this paper, we examine the responses of sheet flow sediment transport under unsteady forcing using a two-phase sheet flow model [Hsu et al., 2004]. We demonstrate that whether the sediment transport rate can be successfully parameterized by solely the magnitude of free-stream velocity strongly depends on the wave shape. We find that in general the time-dependent sediment transport is highly coherent with the corresponding instantaneous bed shear stress. However, for certain wave shapes, the time evolution of bed shear stress cannot be parameterized by the instantaneous free-stream velocity in a quasisteady sense.

[5] After a brief description of the model, supplementary model validations on the temporal variation of sediment transport rate are presented. To demonstrate that the sediment transport process sometimes cannot be fully described by solely the magnitude of free-stream velocity, we first use the model to examine sheet flow under a prescribed freestream velocity time history of a saw-tooth shape. These results are further interpreted through an examination of the sediment-phase momentum equations to study the relevant physical mechanisms responsible for our observation and to emphasize the effect of horizontal pressure gradient in modeling unsteady sediment dynamics. We then investigate skewed waves typical in shallow water outside the surf zone. To further explore and quantify the relation between bed failure and sediment transport, the sediment transport is next forced by using a free-stream velocity time history shaped like a ramp, which drives the flow with a constant acceleration between two steady state conditions. Finally, motivated by the results of the two-phase model, we suggest new directions toward an improved parameterization for nearshore sediment transport under sheet flow conditions.

\section{Model Description}

\subsection{Two-Phase Equations}

[6] The two-phase model of Hsu et al. [2004] will be briefly summarized here for the convenience of the reader. We treat the mixture of grains and water as a two-phase mixture where each phase obeys the basic conservation of mass, momentum, and energy. The fluid phase is treated as an incompressible liquid with mass density $\rho^{f}$, and the particle phase is treated as identical spheres with diameter $d$ and mass density $\rho^{s}$. The overall combined sheet flow is considered as that in a U-tube, in which the flow is assumed to be uniform in the flow direction and driven by a prescribed time history of free-stream velocity through the horizontal pressure gradient.

[7] The dynamics of the 1-dimensional fluid-granular flow under consideration are assumed to be governed by the two-phase conservation equations of fluid and sediment. The fluid and sediment phase continuity equations are

$$
\frac{\partial \rho^{f}(1-\bar{c})}{\partial t}+\frac{\partial \rho^{f}(1-\bar{c}) \tilde{w}^{f}}{\partial z}=0
$$

$$
\frac{\partial \rho^{s} \bar{c}}{\partial t}+\frac{\partial \rho^{s} \bar{c} \tilde{w}^{s}}{\partial z}=0,
$$

where $z$ is normal to the channel bottom and $\tilde{w}^{f}$ and $\tilde{w}^{s}$ are, respectively, the $z$-components of the fluid and particle velocities.

[8] The horizontal $(x)$ and vertical $(z)$ components of the fluid-phase momentum equations can be expressed as

$$
\begin{aligned}
\frac{\partial \rho^{f}(1-\bar{c}) \tilde{u}^{f}}{\partial t}= & -\frac{\partial \rho^{f}(1-\bar{c}) \tilde{u}^{f} \tilde{w}^{f}}{\partial z}-(1-\bar{c}) \frac{\partial \bar{P}^{f}}{\partial x} \\
& +\frac{\partial \tau_{x z}^{f}}{\partial z}-\beta \bar{c}\left(\tilde{u}^{f}-\tilde{u}^{s}\right) \\
\frac{\partial \rho^{f}(1-\bar{c}) \tilde{w}^{f}}{\partial t}= & -\frac{\partial \rho^{f}(1-\bar{c}) \tilde{w}^{f} \tilde{w}^{f}}{\partial z}-(1-\bar{c}) \frac{\partial \bar{P}^{f}}{\partial z}+\frac{\partial \tau_{z z}^{f}}{\partial z} \\
& +\rho^{f}(1-\bar{c}) g-\beta \bar{c}\left(\tilde{w}^{f}-\tilde{w}^{s}\right)+\beta v_{f t} \frac{\partial \bar{c}}{\partial z}
\end{aligned}
$$

where $\tilde{u}_{f}$ and $\tilde{u}_{s}$ are, respectively, the $x$-components of the fluid and particle velocities, $\bar{P}^{f}$ is the fluid pressure, $\tau_{x z}^{f}$ and $\tau_{z z}^{f}$ are fluid phase stresses, including the fluid viscous stress and the fluid Reynolds stresses, and $g=-9.8\left(\mathrm{~m} / \mathrm{s}^{2}\right)$ is the gravitational acceleration. The last two terms in equation (4) are the Favre-averaged [e.g., Drew, 1976] drag force with $\beta$ defined as

$$
\beta=\frac{\rho^{f} U_{r}}{d}\left(\frac{18.0}{R e_{p}}+0.3\right) \frac{1}{(1-\bar{c})^{n}},
$$

in which

$$
U_{r}=\sqrt{\left(\tilde{u}^{f}-\tilde{u}^{s}\right)^{2}+\left(\tilde{w}^{f}-\tilde{w}^{s}\right)^{2}}
$$

is the magnitude of the relative velocity between the fluid and sediment phase, and $R e_{p}=\rho^{f} U_{r} d / \mu_{f}$ is the particle Reynolds number, with $\mu_{f}$ the fluid viscosity. In equation (5) the experimental results of Richardson and Zaki [1954] are adopted to incorporate the effect of sediment concentration on the drag force, with $n$ a coefficient depending on the particle Reynolds number,

$$
n=4.45 R e_{p}^{-0.1}-1, \quad 1 \leq R e_{p}<500 .
$$

The last term in equation (4) is the modeled form for fluid turbulent suspension based on gradient transport, with $v_{f t}$ the fluid eddy viscosity.

[9] The $x$ and $z$ components of the sediment-phase momentum equations are

$$
\begin{gathered}
\frac{\partial \rho^{s} \bar{c} \tilde{u}^{s}}{\partial t}=-\frac{\partial \rho^{s} \bar{c} \tilde{u}^{s} \tilde{w}^{s}}{\partial z}-\bar{c} \frac{\partial \bar{P} f}{\partial x}+\frac{\partial \tau_{x z}^{s}}{\partial z}+\beta \bar{c}\left(\tilde{u}^{f}-\tilde{u}^{s}\right) \\
\frac{\partial \rho^{s} \bar{c} \tilde{w}^{s}}{\partial t}=-\frac{\partial \rho^{s} \bar{c} \tilde{w}^{s} \tilde{w}^{s}}{\partial z}-\bar{c} \frac{\partial \bar{P}^{f}}{\partial z}+\frac{\partial \tau_{z z}^{s}}{\partial z} \\
+\rho^{s} \bar{c} g+\beta \bar{c}\left(\tilde{w}^{f}-\tilde{w}^{s}\right)-\beta v_{f t} \frac{\partial \bar{c}}{\partial z}
\end{gathered}
$$

where $\tau_{x z}^{s}$ and $\tau_{z z}^{s}$ are the stresses of the sediment phase, including the small-scale particle (intergranular) stresses 
and the Reynolds stresses of the Favre-averaged particle velocities. Note that fluid and sediment phases exchange momentum with each other through the equal and oppositely signed drag terms, and overall momentum is conserved.

\subsection{Closure of Stresses}

[10] The closures of the fluid Reynolds stresses and the sediment stresses are the essential components of the present two-phase sheet flow model. The fluid Reynolds stresses are calculated by the eddy viscosity, which is further calculated by the fluid turbulence kinetic energy $k_{f}$ and its dissipation rate $\epsilon_{f}$. The balance equations of $k_{f}$ and $\epsilon_{f}$ are derived from the two-phase theory that incorporates essential influences from the sediment phase. Because the present $k_{f}-\epsilon_{f}$ formulations are already presented in detail by Hsu et al. [2003, 2004], they are not repeated here.

[11] To calculate the sediment stress, a measure of the strength of the particle velocity fluctuations,

$$
K_{s}=\frac{1}{2 \bar{c}} \overline{c \Delta u_{i}^{s} \Delta u_{i}^{s}}
$$

the particle fluctuation energy, is introduced. This term is the particle phase analog to turbulent kinetic energy in the fluid phase. The particle fluctuation energy is then calculated by its balance equation,

$$
\begin{aligned}
\rho^{s}\left(\frac{\partial \bar{c} K_{s}}{\partial t}+\frac{\partial \bar{c} K_{s} \tilde{w}^{s}}{\partial z}\right)= & \tau_{x z}^{s} \frac{\partial \tilde{u}^{s}}{\partial z}+\tau_{z z}^{s} \frac{\partial \tilde{w}^{s}}{\partial z}-\frac{\partial Q}{\partial z}-\gamma \\
& +2 \beta \bar{c}\left(\alpha k_{f}-K_{s}\right),
\end{aligned}
$$

with $Q$ as the flux of the fluctuation energy and $\gamma$ as the rate of dissipation. The last term in equation (10) represents the effect of the drag force, with $\alpha$ representing the degree of correlation between the fluid velocity fluctuations and particle velocity fluctuations,

$$
\alpha=\frac{1}{1+T_{p} / \min \left(T_{L}, T_{c}\right)}
$$

with $T_{L}=0.165 k_{f} / \epsilon_{f}$ the fluid turbulence timescale [Elghobashi and Abou-Arab, 1983] and $T_{p}=\rho^{s} / \beta$ the particle response time [Drew, 1976], which measures the time to accelerate a single particle from rest to the velocity of surrounding fluid. The time between collisions $T_{c}=l_{c} / K_{s}^{1 / 2}$ is estimated based on the strength $K_{s}^{1 / 2}$ of sediment velocity fluctuations and the mean free path $l_{c}=\frac{\sqrt{\pi} d}{24 \bar{c} g_{0}(\bar{c})}$ of colliding particles. Here $g_{0}(\bar{c})$ is the contact value of the radial distribution function [Torquato, 1995; Hsu et al., 2004]. To solve equation (10), we need to further incorporate closures for sediment stresses $\tau_{x z}^{s}$ and $\tau_{z z}^{s}$, the flux of fluctuation energy $Q$, and the rate of dissipation $\gamma$.

[12] Because the governing equations for sheet flow are obtained from two averaging processes at different scales [e.g., Hsu et al., 2003], the closures in equation (10) must incorporate intergranular components due to particle-particle interactions and large-scale components of particle velocity fluctuations induced by fluid turbulence [Young and Leeming, 1997]. Following Jenkins and Hanes [1998], the intergranular interactions are assumed to be dominated by particle collisions and a closure based on kinetic theory of collisional granular flow [e.g., Jenkins and Savage, 1983] is implemented. On the other hand, since the large-scale sediment stress is influenced by fluid turbulence, we adopt a closure similar to the one-equation fluid turbulence model. For the detailed mathematical representations of the closures on $\tau_{x z}^{s}, \tau_{z z}^{s}, Q$ and $\gamma$, the readers are referred to Hsu et al. [2004].

[13] In the highly concentrated region nearest and within the stationary bed, where the sediment concentration is greater than the random loose-packing $\left(c_{*}=57 \%\right)$, particles are in relatively long-term enduring contact. Since the fundamental assumption in the kinetic theory requires that the duration of contact between particles must be much smaller than the time between collisions, we need to modify the kinetic theory when modeling sediment stress in the region where sediment concentration is between random loose-packing and random closepacking $\left(c^{*}=63.5 \%\right)$.

[14] For the particle shear stress, we modify the collisional viscosity in the kinetic theory appropriate to the glass transition and adopt the experimental results of Bocquet et al. [2002], which suggest a much larger value of collisional viscosity than that in the kinetic theory when the sediment concentration is greater than random loose-packing. Hence the particle shear stress in the region of enduring contact is modeled as an extremely viscous granular continuum.

[15] For particle normal stress, an additional component $\tau_{z z}^{s e}$ due to enduring contact is incorporated into $\tau_{z z}^{s}$ when the sediment concentration is greater than random loose-packing. We use the closure for $\tau_{z z}^{s e}$ suggested by Jenkins et al. [1989], who analyzed homogeneously packed, identical spheres in Herzian contact. They proposed a formula for particle normal stress phrased in terms of the average compressive volume strain raised to the $3 / 2$ power. Because the volume strain is not determined in the present model, we need to modify the formula of Jenkins et al. [1989] in terms of sediment concentration,

$$
\tau_{z z}^{s e}=\left\{\begin{array}{lc}
0, & \bar{c}<c * \\
\frac{m}{\pi d^{2}} K(\bar{c}) \bar{c}(\bar{c}-c *)^{\chi} & c * \leq \bar{c} \leq c^{*}
\end{array}\right.
$$

where $m$ depends on material properties given in terms of shear modulus and Poisson ratio of the granular material and $K(\bar{c})$ is the coordination number, a function of sediment concentration, representing average numbers of particles with which a particle is in contact. The specific mathematical formulae of $m$ and $K(\bar{c})$ are given by Hsu et al. [2004], and are not repeated here. The power $\chi$ is considered to be a numerical coefficient in the model. Since $\chi$ is strongly related to the boundary conditions at bed, its value shall be given in the next section.

\subsection{Boundary Conditions}

[16] The present model simulates sediment transport processes spanning the entire region from the stationary, porous sediment bed, where sediment particles are immobile, through the slowly shearing region of enduring particle contact, to the rapid flow region of intense particle collisions, and finally to turbulent suspension. A description of the instantaneous (vertical) location of the bed (ILB), which changes in time according to the external flow forcing, must 

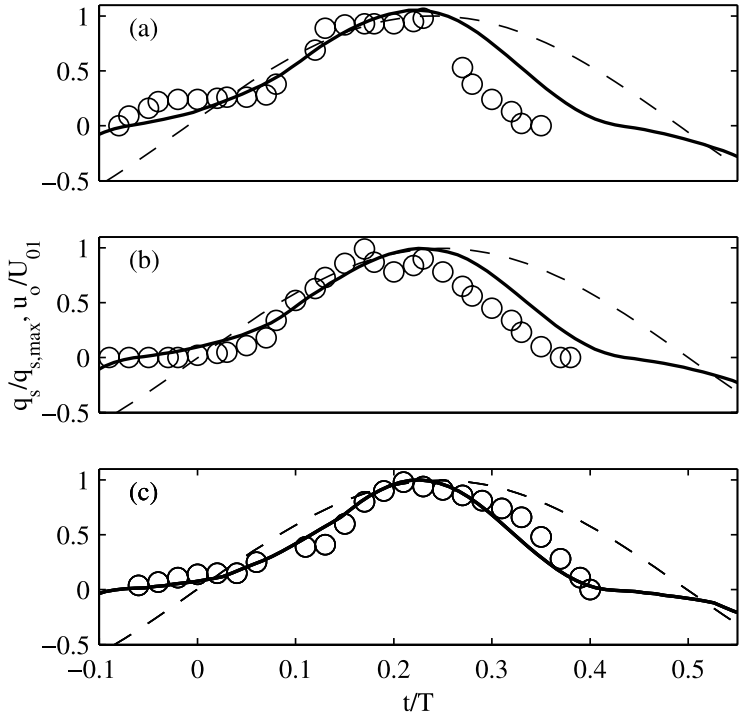

Figure 1. Comparisons of time histories of total sediment transport rate between calculated results (solid curves) and the measured data (symbols) of Asano [1995] using particles of diameter $d=4.17 \mathrm{~mm}$ and specific gravity $s=$ 1.24. The corresponding time histories of free-stream velocity are shown in the dashed curve; (a) case $\mathrm{C} 1$, $U_{01}=0.926 \mathrm{~m} / \mathrm{s}, T=4.64 \mathrm{~s}$; (b) case C2, $U_{01}=0.85 \mathrm{~m} / \mathrm{s}$, $T=4.64 \mathrm{~s}$; and $(3)$ case $\mathrm{C} 4, U_{01}=0.637 \mathrm{~m} / \mathrm{s}, T=4.28 \mathrm{~s}$.

be incorporated. We determine the movement of ILB by incorporating the Coulomb failure criterion,

$$
\tau_{x z}^{s}=\tau_{z z}^{s} \tan \phi,
$$

where $\phi$ is the friction angle of the sediment [see Hanes and Inman, 1985a, 1985b]. We note that through modifying the collisional viscosity to the glass transition, the total bed shear stress becomes part of the solution of the model that must respond to the unsteady forcing. By applying equations (12) and (13) at the stationary bed interface with the known bed shear stress, the corresponding concentration at the bed, which we denote as failure concentration $\hat{c}$, can be calculated. Since the sediment concentration just above the immobile bed must be equal to $\hat{c}$, the location of ILB can then be determined so that the total amount of mobilized sediment is adjusted to satisfy the governing equations of the whole transient two-phase system. In addition, because the value of $\hat{c}$ must be close to but not greater than the random closepacking, an appropriate numerical value for $\chi$ can be calibrated. In the present model, $\chi$ is set to be 5.5, which gives $\hat{c}$ of about $62 \%$ to $63 \%$. Within the porous immobile bed, the sediment mean velocity and fluctuation energy must vanish, and no-slip boundary conditions are specified.

[17] The proposed model is solved numerically with a finite difference scheme. To drive the model with oscillatory flow by a prescribed time history of free stream velocity $u_{0}(t)$, the horizontal pressure gradient is specified according to the acceleration of the free stream velocity,

$$
\frac{1}{\rho^{f}} \frac{\partial \bar{P}^{f}}{\partial x}=-\frac{d u_{o}(t)}{d t} \text {. }
$$

\subsection{Model Validation}

[18] Hsu et al. [2004] validate the model with detailed velocity and concentration profiles from laboratory experiments of Sumer et al. [1996] for steady flow and Asano [1995] for oscillatory flow. In this paper, we are interested in the temporal variation of the volume sediment transport rate,

$$
q_{s}(t)=\int_{0}^{h} \bar{c}(t) \tilde{u}^{s}(t) d z
$$

calculated by integrating the horizontal sediment flux across the water depth $h$ with $z=0$ located below the lowest moving grains. Therefore we present comparisons between the calculated $q_{s}$, with that of Asano [1995] (Figure 1), under the prescribed free-stream velocity time history of a sinusoidal wave,

$$
u_{0}(t)=U_{01} \sin \left(\frac{2 \pi}{T} t\right)
$$

where $U_{01}$ is the velocity amplitude and $T$ is the oscillatory period. The predicted instantaneous sediment transport rates agree fairly well with the measured data. We note here that no specific adjustments of the model parameters are conducted to fit the measured data set. Slightly larger discrepancies can be observed during the settling phase $(t / T=0.22$ to 0.4$)$ for case $\mathrm{C} 1$ and $\mathrm{C} 2$, which is due to the overprediction of sediment velocity. More detailed comparisons for sediment concentration and velocity at various phases are presented by Hsu et al. [2004].

\section{Asymmetric Saw-Tooth Wave Forcing}

[19] The measured time histories of near-bed flow velocity under broken waves often follows a saw-tooth shape [e.g., Elgar and Guza, 1985]. The time history of the sawtooth wave velocity can be described as [e.g., Drake and Calantoni, 2001]

$$
u_{0}(t)=U_{0 s} \sum_{n=1}^{5} \frac{1}{2^{n-1}} \sin \left[n \frac{2 \pi}{T} t+(n-1) \pi\right],
$$

with $U_{0 s}$ the velocity amplitude of the saw-tooth wave. The saw-tooth wave exhibits the following important features: (1) Both the mean and skewness of the saw-tooth wave induced velocity are zero; (2) the flow acceleration is asymmetric with respect to the positive and negative phases (see Figures $2 \mathrm{a}$ and $2 \mathrm{~b}$ ), with large accelerations between trough and crest, and relatively smaller decelerations between crest and trough. Whereas, for a single sinusoidal wave of free-stream velocity (equation (16)), the flow acceleration is symmetric. If sediment transport processes were only dependent on free-stream flow velocity, the magnitude of the sediment transport at the velocity extrema of the saw-tooth wave would be equivalent (though in opposite directions).

[20] The transport of coarse sand of diameter $d=1.1 \mathrm{~mm}$ and specific gravity $s=2.65$ driven by saw-tooth wave forcing (equation (17)) with wave period $T=6.0 \mathrm{~s}$ and amplitude $U_{0 s}=1.0 \mathrm{~m} / \mathrm{s}$ is calculated by the two-phase sheet 

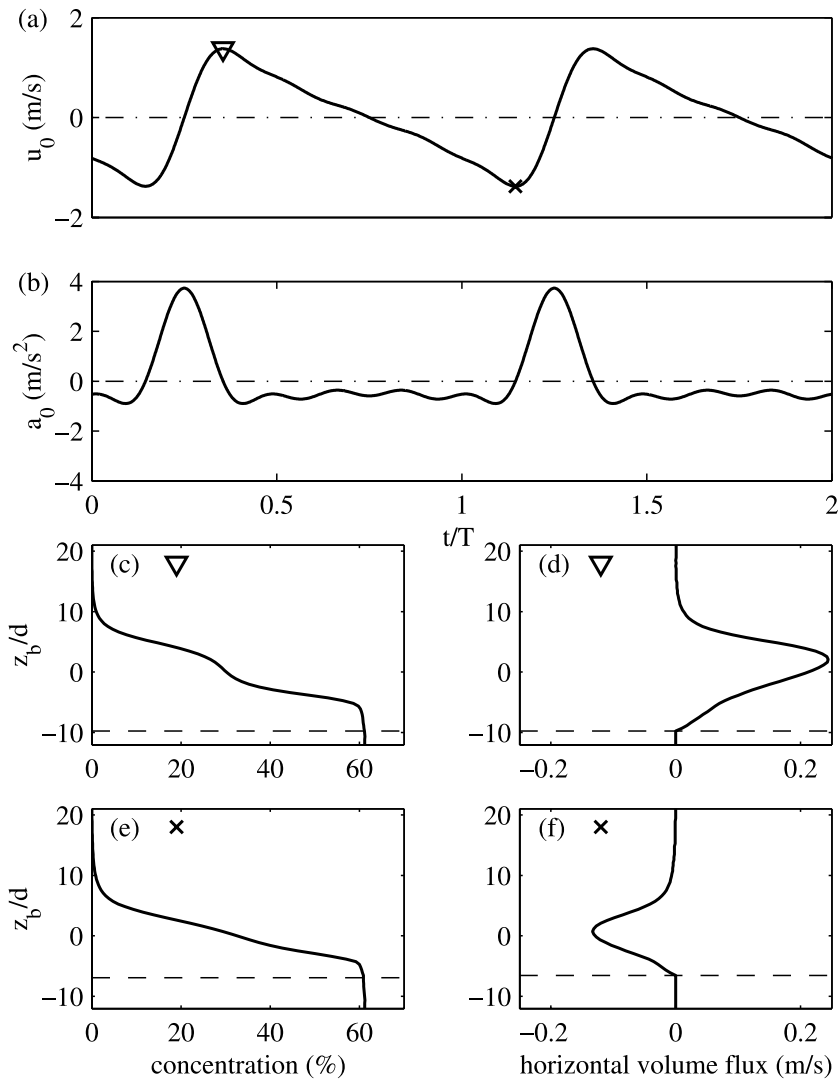

Figure 2. Snapshots of sediment concentration and horizontal volume flux under saw-tooth wave of wave period $T=6.0 \mathrm{~s}$ at the instant of maximum positive freestream velocity (inverted triangle), and maximum negative velocity (cross). (a) Prescribed free-stream velocity, (b) freestream acceleration, (c) sediment concentration, and (d) horizontal volume flux at the inverted triangles; (e) sediment concentration and (f) horizontal volume flux at the crosses.

flow model. The sediment concentration and horizontal sediment flux $\bar{c} \tilde{u}^{S}$ at the instant of maximum positive freestream velocity and that of maximum negative velocity are presented in Figures $2 \mathrm{c}, 2 \mathrm{~d}$ and $2 \mathrm{e}, 2 \mathrm{f}$, respectively. The corresponding time history of the saw-tooth free-stream velocity and acceleration are also shown in Figures 2a and $2 \mathrm{~b}$ for reference. It is evident that even though the magnitudes of the maximum positive and maximum negative free-stream velocity are identical, and the accelerations are both zero, the corresponding sediment concentration profiles are quite different. Specifically, the calculated horizontal sediment flux at the instant of maximum positive free-stream velocity is significantly larger (Figure 2d) than that of the maximum negative (Figure 2f). According to the calculated instantaneous location of the bed, marked by the dashed lines in Figures 2c, 2d and 2e, 2f, the bed is lower under maximum positive velocity, suggesting that more sediment is mobilized under the wave crest than under the wave trough. Here, we further utilize the erosion depth $l_{E}$ [e.g., Zala Flores and Sleath, 1998], defined as the distance between the initially undisturbed bed level and the instantaneous location of the interface between the moving and stationary grains in the bed, to quantify the amount of mobilized sediment. Because the large sediment transport under the wave crest occurs shortly after a duration of large free-stream acceleration, it seems that the transport processes, including the erosion depth, may be correlated to the flow acceleration, though not instantaneously.

[21] Similarly, the calculated sediment concentration and horizontal sediment volume flux at the two instants of flow reversal are shown in Figures 3c, 3d and 3e, 3f. Even when the free-stream velocity vanishes at these two instants, some of the sediment remains mobile. In addition, there is significantly more sediment at the instant of crosses, the time of peak acceleration, than that at the inverted triangles, the time of peak deceleration (which is lower in magnitude). This observation seems to be related to the differences in the duration of the settling phase as well as any mobilizing forces present during this phase (such as the pressure gradient) associated with the instant of the crosses and the inverted triangles. During the settling phase, the magnitude of the flow velocity decreases, the fluid and particle induced suspension mechanisms become weaker, and sediment particles tend to settle toward the bed. It takes some time for both the flow turbulence and intergranular collisions to be completely dissipated and the sediment to entirely settle
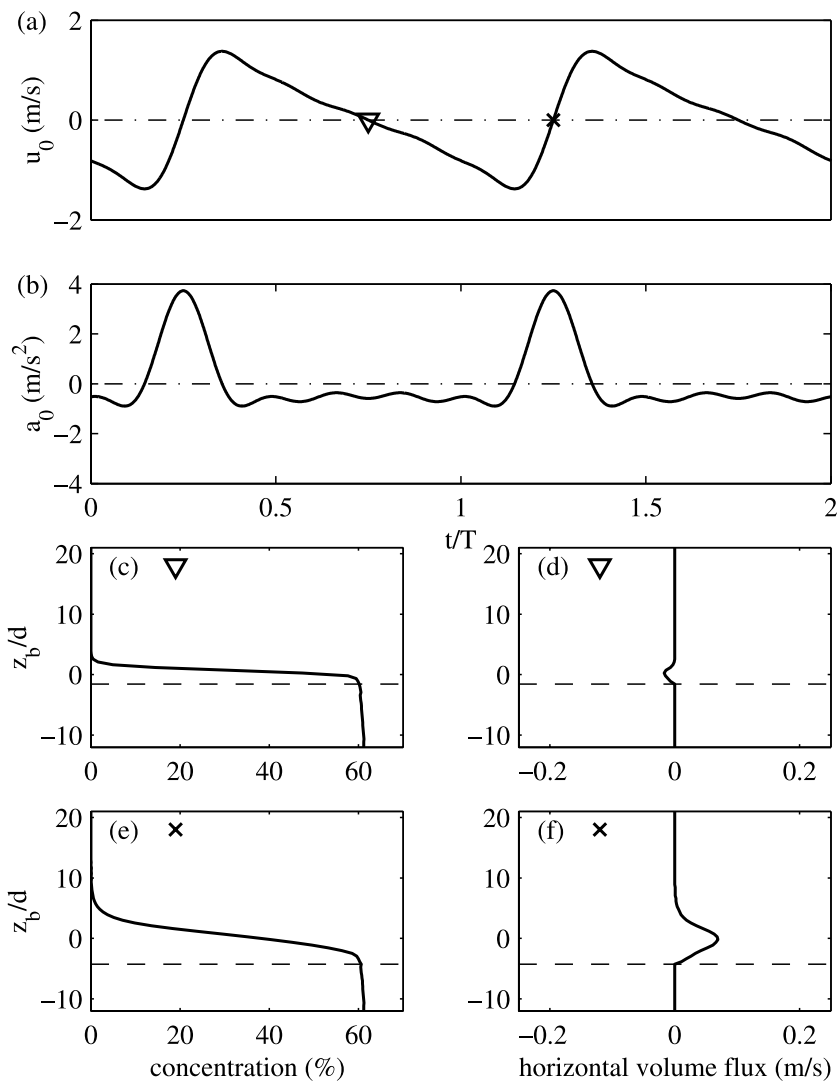

Figure 3. Snapshots of sediment concentration and horizontal volume flux under saw-tooth wave of wave period $T=6.0 \mathrm{~s}$ at two instants of flow reversal. (a) Prescribed free-stream velocity, (b) free-stream acceleration, (c) sediment concentration and (d) horizontal volume flux at the triangles; (e) sediment concentration and (f) horizontal volume flux at the crosses. 

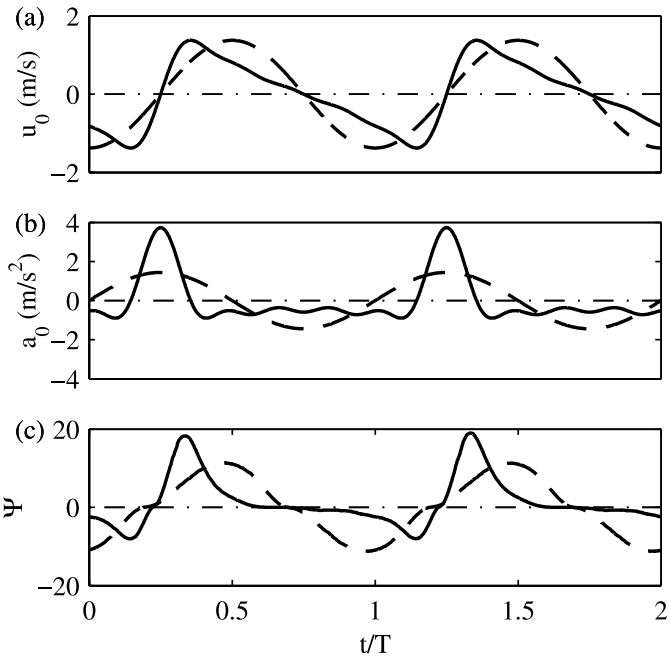

Figure 4. Sediment transport under saw-tooth forcing (solid curve) and single sinusoidal forcing (dashed curve) of wave periods $T=6.0 \mathrm{~s}$. (a) Prescribed free-stream velocity, (b) free-stream acceleration, and (c) nondimensional sediment transport rate.

into the bed. The amount of residual sediment that remains mobilized is less under a shorter duration of the settling phase.

[22] The calculated sediment transport rate under the sawtooth forcing (solid curves), is shown in Figure 4c along with that under single sinusoidal forcing (i.e., equation (16), denoted by the dashed curves) with the same magnitude of RMS velocity and period. The calculated nondimensional volume sediment transport rate is defined as

$$
\Psi(t)=\frac{q_{s}(t)}{\sqrt{(s-1) g d^{3}}} .
$$

In sinusoidal forcing, because both the time history of flow velocity and acceleration are symmetric with respect to the positive and negative phase, the net sediment transport rate is zero, even though the sediment transport rate for each single positive phase or negative phase remains significant. On the other hand, under the saw-tooth forcing, the calculated sediment transport rates for the positive and negative phases are quite different and their magnitudes are not correlated with that of the corresponding free-stream velocity. In fact, their magnitudes are approximately correlated with the corresponding magnitude of free-stream acceleration. This observation is consistent with the results obtained in Figure 2. In particular, the model predicts a sediment transport rate under the positive phase (associated with large acceleration) 2.1 times greater than that under the negative phase (associated with the small acceleration) and a net sediment transport in the positive phase direction is obtained.

[23] The effects of wave period are further investigated by comparing a case of $T=12 \mathrm{~s}$ with the previous results for $T=6 \mathrm{~s}$. Figure $5 \mathrm{~b}$ indicates that longer wave period has a smaller magnitude free-stream acceleration. In Figure 5c, as the overall magnitude of acceleration becomes smaller, the corresponding time history of sediment transport rate asso- ciated with the positive and negative phase becomes more symmetric. This reinforces the speculation that under the saw-tooth wave shape, the flow acceleration is a better parameter (than the flow velocity) for the asymmetric sediment transport rate.

[24] The present results are qualitatively consistent with the discrete element model of Drake and Calantoni [2001], and the hypothesis proposed by Elgar et al. [2001] to explain the observed onshore sandbar migration under near-breaking waves [see also Hoefel and Elgar, 2003]. Because the magnitudes of sediment transport rate cannot be fully explained by the corresponding magnitudes of freestream velocity under saw-tooth waves, we shall further explain the physical mechanisms responsible for this phenomenon next.

[25] In the two-phase theory, the mechanics of sediment transport can be analyzed based on the horizontal sediment flux $\bar{c} \tilde{u}^{S}$ using the horizontal momentum equation of the sediment phase (equation (7)). According to the right-hand side of equation (7), several mechanisms contribute to the temporal variations in the horizontal sediment flux. They are: the convection, the horizontal pressure gradient (equivalent to the free-stream acceleration in the present model), the vertical gradient of the particle shear stress, and the drag from the fluid phase. A qualitative picture of horizontal sediment flux can be described as follows.

[26] In a fully developed flow, the horizontal particle motion results from several forces. The fluid pressure applies a net force to the sediment particles. This mechanism is represented by the horizontal pressure gradient term in equation (7). In addition, the fluid drag force, represented by the last term in equation (7), contributes to sediment particle motion. Furthermore, due to the presence of stationary bed and the no-slip boundary condition of the particle velocity there, the particle horizontal motion is retarded by the vertical gradient of particle shear stress (the third term in equation (7)) that results from particle-
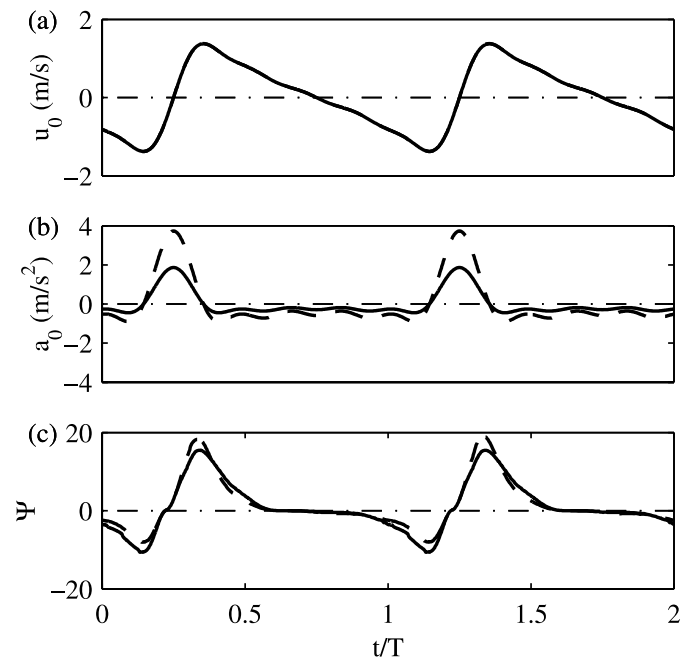

Figure 5. Sediment transport under saw-tooth forcing with $T=12.0 \mathrm{~s}$ (solid curve), and $T=6.0 \mathrm{~s}$ (dashed curve). (a) Prescribed time history of free-stream velocity, (b) freestream acceleration, and (c) nondimensional sediment transport rate. 

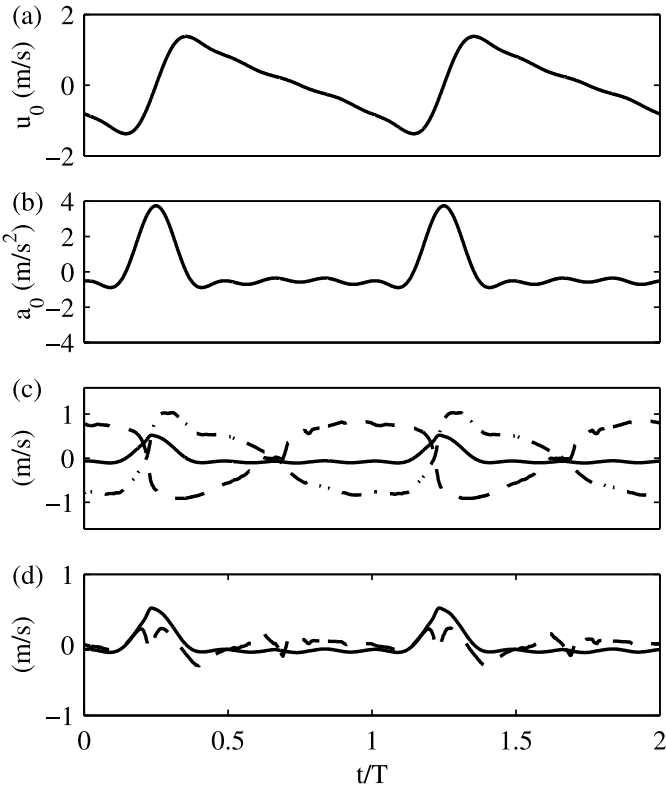

Figure 6. Horizontal sediment momentum equation budget under saw-tooth wave of $T=6.0 \mathrm{~s}$ at initially undisturbed bed level. (a) Free-stream velocity, (b) freestream acceleration, and (c) horizontal pressure gradient (solid curve), drag (dash-dotted curve), vertical gradient of particle stress (dashed curve). (d) Sum of drag and vertical gradient of particle stress (dashed curve) versus horizontal pressure gradient (solid curve).

particle interactions (e.g., collisions). Finally, the vertical motion of particles also induces exchange of horizontal momentum through vertical convection (the first term in equation (7)).

[27] It is not difficult to carry out an order-of-magnitude estimate on the relative magnitude of these effects in equation (7). On the basis of a first-order boundary layer approximation, the convection term can be neglected. Since the drag (quadratic) term is proportional to the area of the particle $\left(\sim d^{2}\right)$ while the pressure gradient term is proportional to the volume of the particle $\left(\sim d^{3}\right)$, the magnitude of the drag force shall be significantly larger than that of the horizontal pressure gradient force for typical sediment sizes. Nevertheless, the pressure gradient term may still be important during a short interval near flow reversal when the flow velocity vanishes. Therefore, despite the fact that the particle stress in the present problem cannot be simply estimated using dimensional considerations, one may conclude that the flow acceleration associated with the horizontal pressure gradient force will be, in general, not as important as that associated with the drag force.

[28] To understand the role of particle stress in equation (7) and further estimate its magnitude, we utilize the detailed descriptions in sheet flow model. Figure $6 \mathrm{c}$ presents the time history of the horizontal pressure gradient term, the particle stress term, and the drag term associated with equation (7) at the initially undisturbed bed level (i.e., $z_{b} / d=0$ ) under the saw-tooth wave forcing. Since the magnitude of convection term is indeed small, it is not shown here for convenience. Notice that the location $z_{b}=$ 0 evaluated in Figure $6 \mathrm{c}$ corresponds to the concentrated region of the sheet where the sediment concentration is no less than $30 \%$ during the entire wave. Comparing the horizontal pressure gradient (solid curve) and the drag (dash-dotted curve), the magnitude of the pressure gradient term is generally smaller than that of the drag term and is only important during a short instant of flow reversal. This is consistent with the previous order-of-magnitude estimate. However, comparing the drag (dash-dotted curve) and the particle stress (dashed curve), the magnitude of particle stress term is comparable to that of the drag term but opposite in sign. In other words, the particle stress term is effectively balancing the drag term over the entire wave cycle. To further demonstrate this feature, Figure $6 \mathrm{~d}$ presents the sum of the particle stress term and the drag term, represented by the dashed curve. The corresponding pressure gradient term (solid curve) is shown again for reference. Although the individual magnitudes of the pressure gradient term may not be significant compared with the drag term, nevertheless, the presence of the particle stress term due to particle intergranular interactions balances with the drag. Consequently, the horizontal pressure gradient remains important in equation (7). Moreover, examining the drag term itself, because the relative difference between the fluid velocity and particle velocity becomes larger during the phase of large flow acceleration near flow reversal, the magnitude of the drag term is enhanced and causes the sum of the particle stress and drag terms (dashed curve in Figure 6d) to have two peaks of the same sign as the horizontal pressure gradient term.

[29] Figure 7 presents the time history of the horizontal sediment momentum equation budget at five grain diameters above the initially undisturbed bed level (i.e., $z_{b} / d=5$ ).
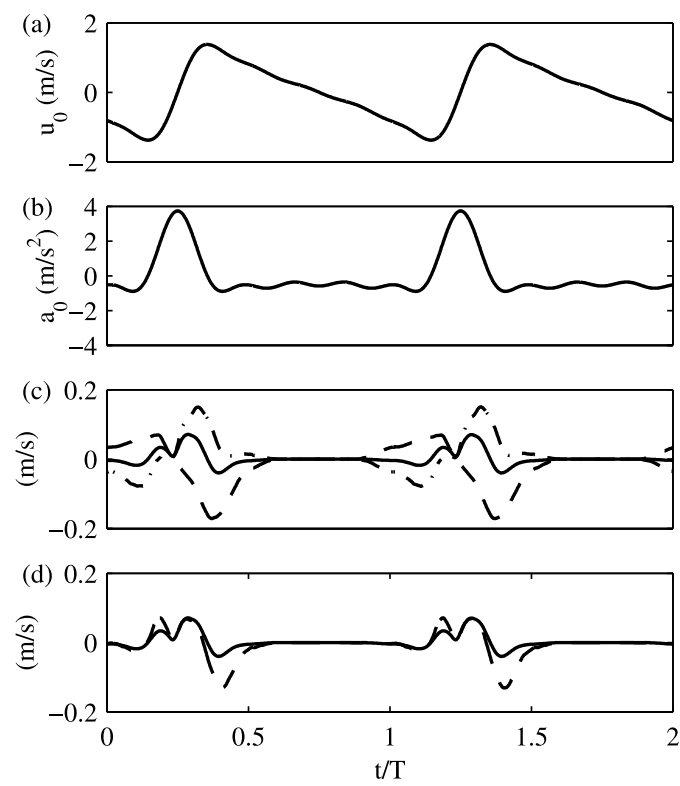

Figure 7. Horizontal sediment momentum equation budget under saw-tooth wave of $T=6.0 \mathrm{~s}$ at $z_{b} / d=5$. (a) Free-stream velocity, (b) free-stream acceleration, and (c) horizontal pressure gradient (solid curve), drag (dash-dotted curve), vertical gradient of particle stress (dashed curve). (d) Sum of drag and vertical gradient of particle stress (dashed curve) versus horizontal pressure gradient (solid curve). 
Since the sediment concentration at this location is much less than that of Figure 5 (no more than $15 \%$ during an entire wave), the overall magnitude of the terms in equation (7) are also smaller. Here we start to notice some imbalance between the particle stress and drag. In Figure 7d, the direct effect of horizontal pressure gradient is less significant than in the more concentrated region. Two peaks (dashed curve) still appear during the flow reversal; it seems that the effect of the larger velocity difference between the two phases during the flow reversal becomes relatively more important.

[30] Because the balance between the vertical gradient of particle stress and the drag determines a major part of the mechanics in the concentrated sheet, further investigation on this feature is warranted. In a steady, fully developed sheet flow, the mean vertical particle velocity vanishes and equation (7) can be further simplified into

$$
-\bar{c} \frac{\partial \bar{P}^{f}}{\partial x}+\frac{\partial \tau_{x z}^{s}}{\partial z}+\left|\beta \bar{c}\left(\tilde{u}^{f}-\tilde{u}^{s}\right)\right|=0 .
$$

To estimate the magnitude of the horizontal pressure gradient in a steady state condition, we rephrase the horizontal pressure gradient in terms of the friction velocity, $u *$, and an equivalent water depth, $h$,

$$
\frac{\partial \bar{P}^{f}}{\partial x}=\frac{\rho^{f} u_{*}^{2}}{h} .
$$

By further assuming that the difference between the fluid velocity and particle velocity is on the order of the friction velocity, the relative magnitude between the horizontal pressure gradient and the drag in equation (19) can be estimated as

$$
\frac{\left|\bar{c} \quad \partial \bar{P}^{f} / \partial x\right|}{\left|\beta \bar{c}\left(\tilde{u}^{f}-\tilde{u}^{s}\right)\right|} \approx \frac{T_{p} u_{*}}{s h}
$$

[31] For sand of diameter $1.1 \mathrm{~mm}$, the particle response time, $T_{p}=\rho^{s} / \beta$, is on the order of $0.01 \mathrm{~s}$. In a typical open channel flow, the ratio between the friction velocity and water depth is smaller than order 1 . Therefore the magnitude of the horizontal pressure gradient shall be in general much smaller than that of the drag, and the equilibrium in equation (19) must be established under the condition that the particle stress is approximately balanced with the drag.

[32] Next, consider the equilibrium to be destroyed due to a sudden acceleration of the flow. The timescales of both the drag and particle stress to adjust to the perturbation need to be estimated. For the drag term, this timescale is simply the particle response time $T_{p}$. The timescale for particle stress term is estimated from the diffusion process based on the kinetic theory of collisional grain flow. Since the magnitude of the sediment diffusivity is in general very large due to the high collisional frequency in the concentrated region (i.e., proportional to the contact value of the radial distribution function), the diffusion timescale of particle stress is expected to be very small in the concentrated sheet. On the other hand, the timescale of the free-stream forcing (e.g., the wave period) is in general much larger than either the particle response time or diffusion timescale of particle stress. Consequently, the drag term and particle stress term in equation (19) will adjust themselves almost immediately to the free-stream forcing and remain in balance with each other, approximately. This provides a qualitative explanation for the reason for the approximate balance between the gradient of the particle stress and drag even under unsteady conditions.

[33] In summary, by considering the particle intergranular interactions and fluid-particle momentum transfer using two-phase theory, the particle stress and drag dominate but also counteract each other in the overall horizontal sediment momentum budget. This provides a plausible explanation for why horizontal pressure gradient, or equivalently, flow acceleration has a demonstrable effect on the sediment-phase dynamics under conditions considered here.

\section{Skewed Wave Forcing}

[34] A typical shoaled but unbroken wave in the nearshore exhibits skewness, with the crest exhibiting a greater amplitude and shorter duration than the trough. For convenience, we choose the time history of the skewed wave freestream velocity prescribed by that of the Stokes 2 nd order wave,

$$
u_{0}(t)=U_{01} \sin \left(\frac{2 \pi}{T} t\right)+U_{02} \sin \left(\frac{4 \pi}{T} t\right)
$$

with $U_{01}$ and $U_{02}$ the velocity amplitude associated with the first and the second harmonics of the wave. In the skewed wave, because both the flow velocity and flow acceleration under the positive phase are significantly larger than that under the negative phase, a net sediment transport to the positive wave direction is expected.

[35] The calculated sediment concentration and horizontal sediment volume flux $\bar{c} \tilde{u}^{S}$ at the instant of maximum positive free-stream velocity and that at the maximum negative velocity for the same sediment considered earlier are presented in Figure 8. To match the same magnitude of the RMS velocity to that of the saw-tooth wave presented in the previous section, $U_{01}=1.12 \mathrm{~m} / \mathrm{s}, U_{02}=0.28 \mathrm{~m} / \mathrm{s}$, and $T=6.0 \mathrm{~s}$ are specified. The resulting velocity time history has a velocity skewness of 0.48 , which is high but not unreasonable compared to field observations [e.g., Doering et al., 2000]. In Figures 8c and 8d, it is evident that under the maximum positive phase, a significant amount of sediment is transported. In contrast, under the maximum negative phase (see Figures 8e and 8f), a significantly smaller amount of sediment remains mobile, and the erosion depth $l_{E}$ is only about five grain diameters.

[36] In Figure 9, the calculated sediment concentration and sediment volume flux at the two instants of flow reversal are shown. Again, a noticeable amount of sediment remains mobile when the free-stream velocity vanishes. In addition, comparing between Figures $9 \mathrm{~d}$ and $9 \mathrm{f}$, unlike the corresponding results (Figure 3) for the saw-tooth wave condition, the magnitude of the sediment flux at the two instants of flow reversal are quite close in magnitude, though oppositely directed.

[37] The calculated time history of the sediment transport rate (Figure 10, solid curve) indicates that total sediment transport rate under the positive phase of the skewed wave is 1.4 times larger than that under negative phase. A case of single sinusoidal forcing of the same magnitude of RMS 

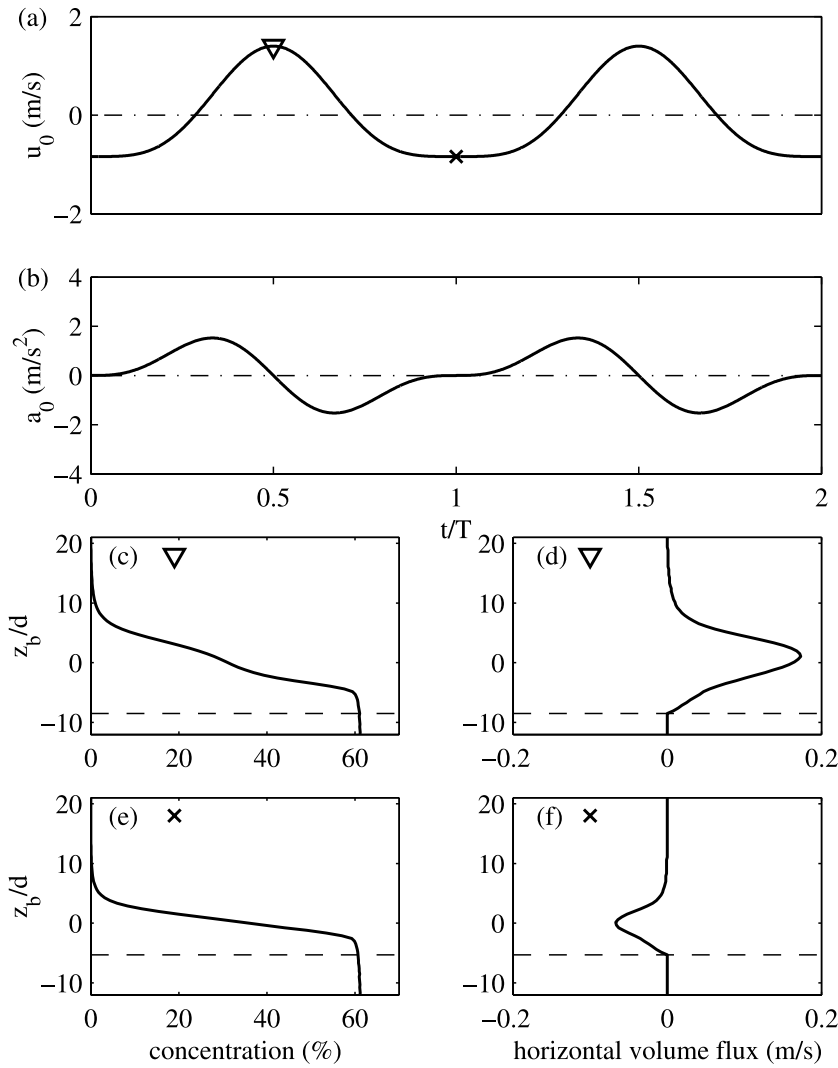

Figure 8. Snapshots of sediment concentration and horizontal volume flux under Stokes second-order wave of wave period $T=6.0 \mathrm{~s}$ at the instant of maximum positive free-stream velocity (inverted triangles), and maximum negative velocity (crosses). (a) Prescribed free-stream velocity, (b) free-stream acceleration, (c) sediment concentration, and (d) horizontal volume flux at the inverted triangles; (e) sediment concentration and (f) horizontal volume flux at the crosses.

velocity is also plotted here for reference. From a parameterization point of view, under the present skewed wave shape, the net sediment transport rate may be plausibly described by the free-stream flow velocity. However, on the basis of the shape of the sediment transport rate time history, we observe that within the positive or negative phase, a larger magnitude of sediment transport tends to bulge toward the times of larger magnitude of accelerations. This is especially obvious during the negative phase where the magnitude of flow velocity is relatively small for the skewed wave. For example, in Figure 10c, the peak magnitude of negative sediment transport occurs at about $t / T=$ 0.81 , while the peak magnitude of free-stream velocity and free-stream acceleration in the negative phase occurs at $t / T=$ 1.0 and $t / T=0.67$, respectively; suggesting that sediment transport is influenced by flow acceleration. Despite the detailed instantaneous variation, the overall feature in the skewed wave is that the magnitudes of free-stream velocity and corresponding acceleration are well correlated (i.e., the times of large velocity are close to the times of large acceleration and vise versa.). Therefore it has been reported that the sediment transport rate under skewed waves can be successfully parameterized by the third moment of freestream velocity [e.g., Ribberink, 1998].

\section{Step Acceleration Forcing}

[38] Various sediment responses between two steady states using a prescribed free stream velocity of a ramp shape are now investigated to further examine the flow acceleration (or equivalently, the horizontal pressure gradient) effect. First, we investigate the transient response between two steady state conditions, where the mixture boundary layer flow slowly adjusts itself from a lower freestream velocity to a higher free-stream velocity due to an instantaneous jump in the pressure gradient applied to force the system. Next, a large pressure gradient is prescribed during a finite interval between the two steady states such that a constant acceleration of the free stream velocity is established during the transient.

[39] Figure 11 illustrates the transport of coarse sand (diameter $d=1.1 \mathrm{~mm}$, specific gravity $s=2.65$ ) during the transient between a low free-stream velocity of $1.02 \mathrm{~m} / \mathrm{s}$ to a high free-stream velocity of $2.18 \mathrm{~m} / \mathrm{s}$ due to an instantaneous increase in the pressure gradient applied at $t=0$. In Figure 11a the flow has already achieved the first steady state of free-stream velocity $1.02 \mathrm{~m} / \mathrm{s}$ before $t=0 \mathrm{~s}$
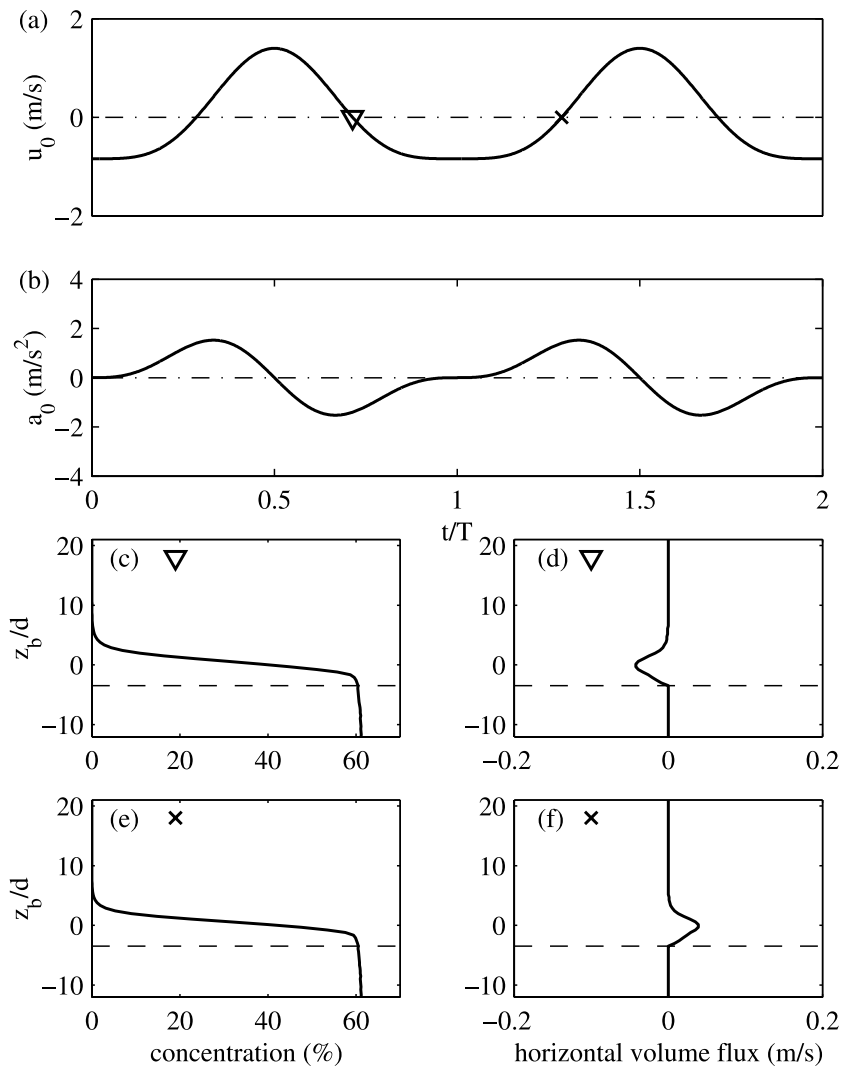

Figure 9. Snapshots of sediment concentration and horizontal volume flux under Stokes second-order wave of wave period $T=6.0 \mathrm{~s}$ at two instants of flow reversal. (a) Prescribed free-stream velocity, (b) free-stream acceleration, (c) sediment concentration, and (d) horizontal volume flux at the inverted triangles; (e) sediment concentration and (f) horizontal volume flux at the crosses. 

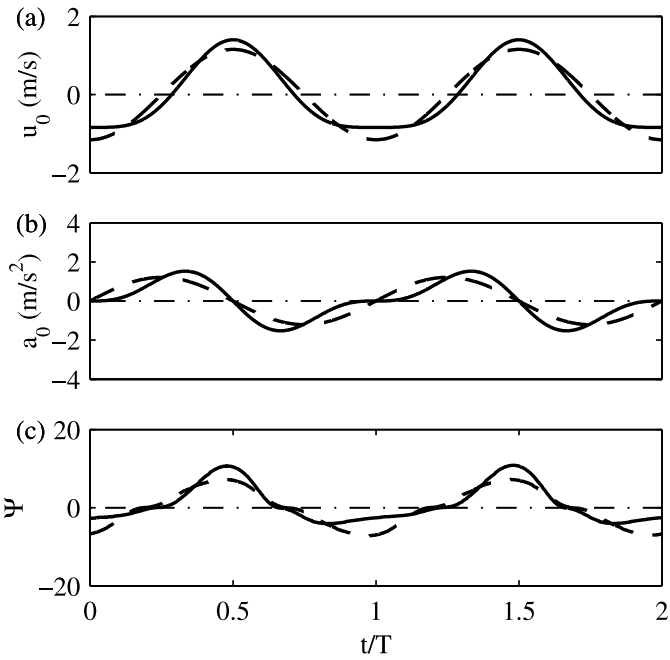

Figure 10. Sediment transport under skewed forcing (solid curve) and single sinusoidal forcing (dashed curve) of wave period $T=6.0 \mathrm{~s}$. (a) Prescribed free-stream velocity, (b) free-stream acceleration, and (c) nondimensional sediment transport rate.

due to a lower horizontal pressure gradient. A higher horizontal pressure gradient is imposed after $t=0 \mathrm{~s}$, and the flow adjusts itself to a second steady state of free-stream velocity $2.18 \mathrm{~m} / \mathrm{s}$. In terms of the free-stream velocity and nondimensional total sediment transport rate (Figures 11a and $11 \mathrm{~b}$ ), it takes about $150 \mathrm{~s}$ for the entire flow (both the fluid and sediment phase) to adjust to the next steady state. On the other hand, according to the erosion depth $l_{E}$ (Figure 11c), it only takes about $10 \mathrm{~s}$ for the bed to reach the location of the next steady state. Therefore the response time for bed failure and sediment entrainment is much shorter than that of the entire boundary layer flow. The bed responds to the variation of the external forcing quickly because the failure/entrainment process occurs very near the bed where sediment concentration is large, particle intergranular interaction dominates, and the diffusion of momentum becomes very efficient. On the contrary, the total sediment transport rate, including some transport through fluid turbulent suspension, must adjust to the external forcing according to the turbulence timescale of the entire flow domain.

[40] Two cases for the transport of coarse sand undergoing a constant acceleration between the two steady states are presented in Figure 12. Between Figures 12b and 12c, the flow is accelerated with a constant flow acceleration of $2.37 \mathrm{~m} / \mathrm{s}^{2}$ from the low free-stream velocity stage of $1.02 \mathrm{~m} / \mathrm{s}$ to the high free-stream velocity stage of $2.18 \mathrm{~m} / \mathrm{s}$ within duration of $\Delta t=0.49 \mathrm{~s}$. Between Figures $12 \mathrm{~b}$ and $12 \mathrm{~d}$, the flow is accelerated between the same limiting velocities within a duration of $\Delta t=1.22 \mathrm{~s}$ due to a lower constant acceleration of $0.95 \mathrm{~m} / \mathrm{s}^{2}$. Figures $12 \mathrm{~b}$ and $12 \mathrm{c}$ show snapshots of the sediment concentration, horizontal sediment flux, and particle and fluid velocity fluctuation intensities under acceleration of $2.37 \mathrm{~m} / \mathrm{s}^{2}$ at the beginning of the acceleration, denoted by the circle, and at the end of the acceleration, denoted by the asterisk, respectively. The dashed curve in Figure 12c corresponds to the results of a steady state case of free stream velocity of $2.18 \mathrm{~m} / \mathrm{s}$. Comparison between the dashed curve and the solid curve illustrates the effect of flow acceleration on sediment transport at the same free-stream velocity. From the sediment flux in snapshots b-2 and c-2, it is evident that within the short duration of $0.49 \mathrm{~s}$, more sediment is mobilized due to higher magnitude of the flow. Specifically, comparing the solid curve and the dashed curve in snapshot c-2, the immobile bed location is significantly lower under large acceleration than that of no acceleration. The flow acceleration considerably enhances the bed failure and mobilizes more sediment. An interesting feature of the newly failed region is that although it is mobile, the sediment concentration remains very large and its vertical distribution is very close to that of the steady state condition. This region could be identified as a highly concentrated plug flow [Sleath, 1999; Foster et al., 2002]. Moreover, from snapshots c-3 and c-4, noticeable particle fluctuations in the newly failed region are observed while the fluid turbulence remains negligible due to high sediment concentration. Snapshots d-1 to d-4 show the corresponding snapshots for the case of lower constant acceleration of $0.95 \mathrm{~m} / \mathrm{s}^{2}$. On the basis of sediment concentration and horizontal flux shown in snapshots $\mathrm{d}-1$ and $\mathrm{d}-2$, the differences are less significant between the case of acceleration of $0.95 \mathrm{~m} / \mathrm{s}^{2}$ and that of no acceleration. The bed locations between these two conditions become very close, suggesting that a flow acceleration of $0.95 \mathrm{~m} / \mathrm{s}^{2}$ cannot cause significant bed failure for the present particles.

[41] It appears that bed failure is responsible for a major part of the excessive sediment transport under large accelerations. We next conduct further investigations on the relation between the flow acceleration, the bed failure, and the corresponding sediment transport rate using a series of step acceleration forcings. Figure 13 shows the results
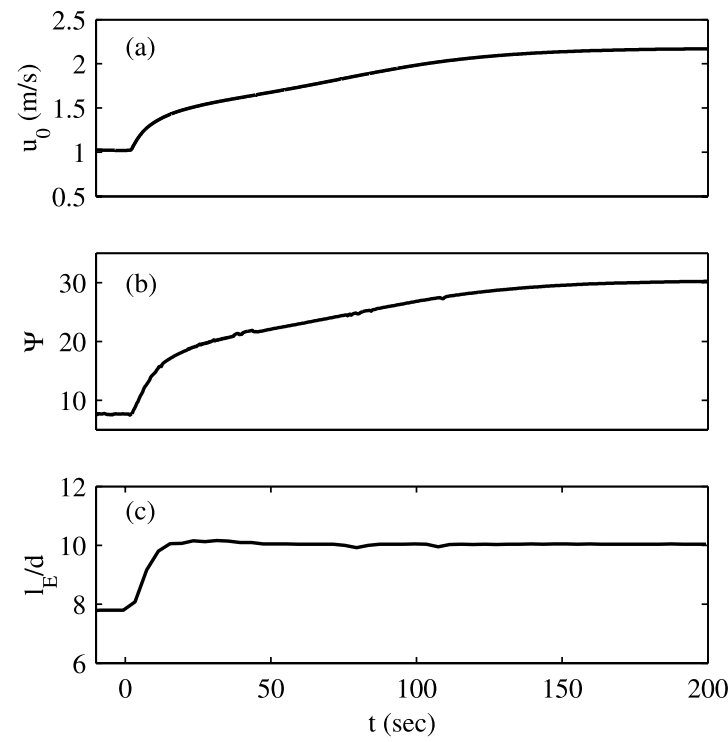

Figure 11. Transport of coarse sand during the transient between a low free-stream velocity of $1.02 \mathrm{~m} / \mathrm{s}$ to a high free-stream velocity of $2.18 \mathrm{~m} / \mathrm{s}$. (a) Time history of freestream velocity, (b) nondimensional sediment transport rate, and (c) nondimensional erosion depth. 


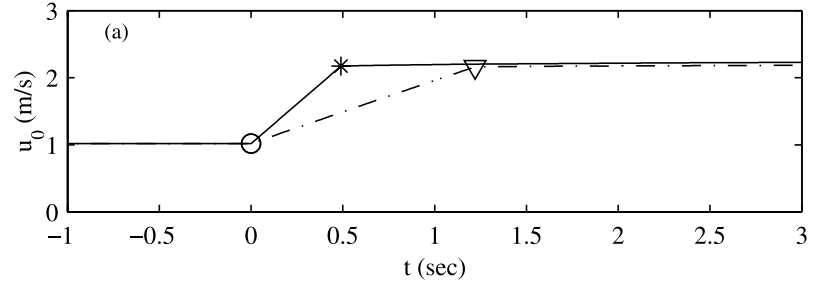

(b)
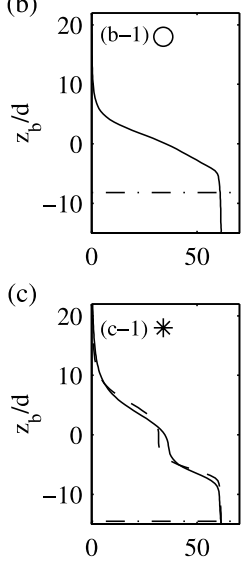

(d)
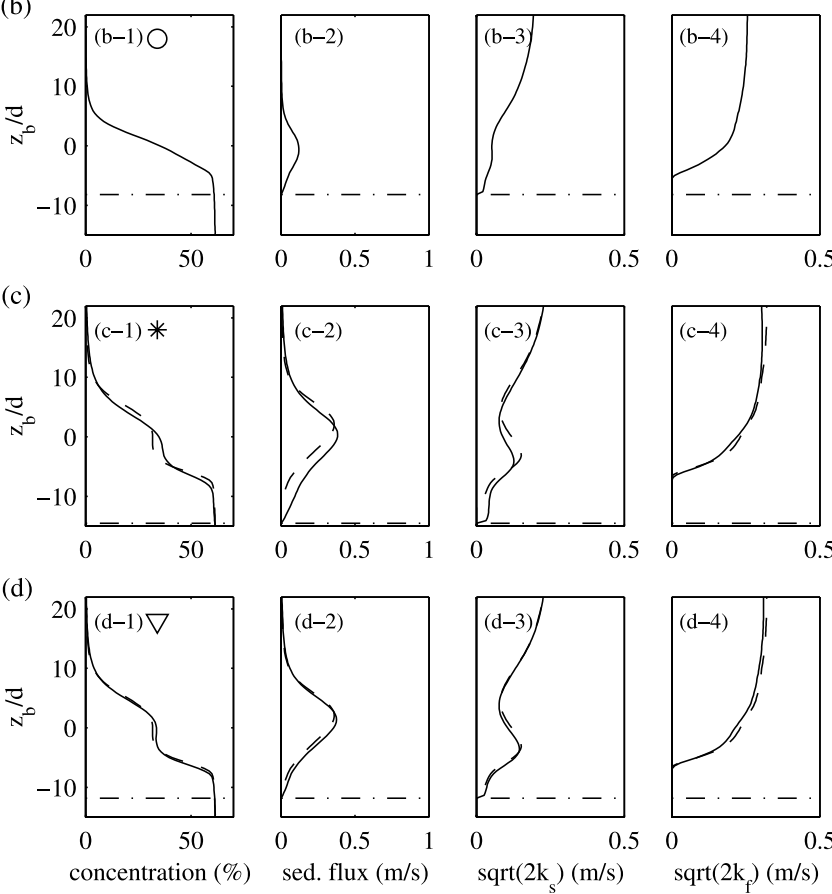

Figure 12. Snapshots of sediment concentration, sediment horizontal flux, and intensity of particle and fluid velocity fluctuations under two constant accelerations of $2.37 \mathrm{~m} / \mathrm{s}^{2}$ (solid curve in Figure 12a) and $0.95 \mathrm{~m} / \mathrm{s}^{2}$ (dash-dotted curve in Figure 12a) of step shapes. In Figures $12 b-12 d$, the solid curves indicate the corresponding results at the circle, asterisk, and inverted triangle shown in Figure 12a, the dashed curves indicate steady state results with the same free-stream velocity of that at the asterisk and inverted triangle, and the dash-dotted lines represent the location of bed.

obtained after forcing the free-stream flow from a low velocity $(1.02 \mathrm{~m} / \mathrm{s})$ to a high velocity (either 1.5 or $2.18 \mathrm{~m} / \mathrm{s}$ ) at various constant accelerations. The sediment transport rates obtained upon just reaching the high freestream velocity of $2.18 \mathrm{~m} / \mathrm{s}$, normalized by its steady state value, are plotted in the solid curve of Figure 13a. The acceleration does not seem to noticeably enhance sediment transport until its magnitude is greater than about $0.25 \mathrm{~m} / \mathrm{s}^{2}$. Within the range of acceleration between $0.25 \mathrm{~m} / \mathrm{s}^{2}$ and $1.0 \mathrm{~m} / \mathrm{s}^{2}$, the enhanced sediment transport rate gradually increases but remains within $10 \%$ of that under the steady state condition. In contrast, as the acceleration becomes greater than about $1.0 \mathrm{~m} / \mathrm{s}^{2}$, the corresponding sediment transport rate increases abruptly to about $30 \%$ more. The major reason for the sudden increase of sediment transport rate is revealed by examining the erosion depth $l_{E}$. In the solid curve of Figure 13b, the erosion depth is made nondimensional by the grain diameter and plotted against the acceleration. Evidently, as the acceleration becomes greater than about $1.0 \mathrm{~m} / \mathrm{s}^{2}, l_{E}$ increases abruptly from about 10 grain diameters to about 15 grain diameters. This results in the large sediment transport rate observed in Figure 13a. Therefore, according to the present model, there seems to exist a critical acceleration for given sediment particle properties such that severe failure occurs across a significant depth of the sediment bed.

[42] Somewhat different behavior is obtained as we evaluate the sediment transport rate at the instant where free-stream velocity just reaches a smaller magnitude of $1.5 \mathrm{~m} / \mathrm{s}$ (dotted curves of Figure 13). Since both the results in the solid curve and dotted curve series are due to the same magnitude of low stage free-stream velocity, evaluating at a lower high free-stream velocity also corresponds to a shorter duration of the constant acceleration. Nevertheless, because the calculated sediment transport rate for each case in Figure 13a has already been normalized by its corresponding sediment transport rate in the steady state, we believe that the observed differences between the solid curve series and the dotted curve series are due to the differing durations of acceleration rather than due to the different velocities. Specifically, when evaluated at a smaller high free-stream velocity of $1.5 \mathrm{~m} / \mathrm{s}$ (dotted curve), a constant acceleration with a magnitude between $0.1 \mathrm{~m} / \mathrm{s}^{2}$ and $2.0 \mathrm{~m} / \mathrm{s}^{2}$ causes the corresponding sediment transport rate to be smaller than that in the steady state (i.e., $q_{s} / q_{s}$ smaller than unity). This is because when acceleration is small, the corresponding horizontal pressure gradient is not strong enough to cause bed failure (see the erosion depth plotted in Figure 13b). On the other hand, it takes time for
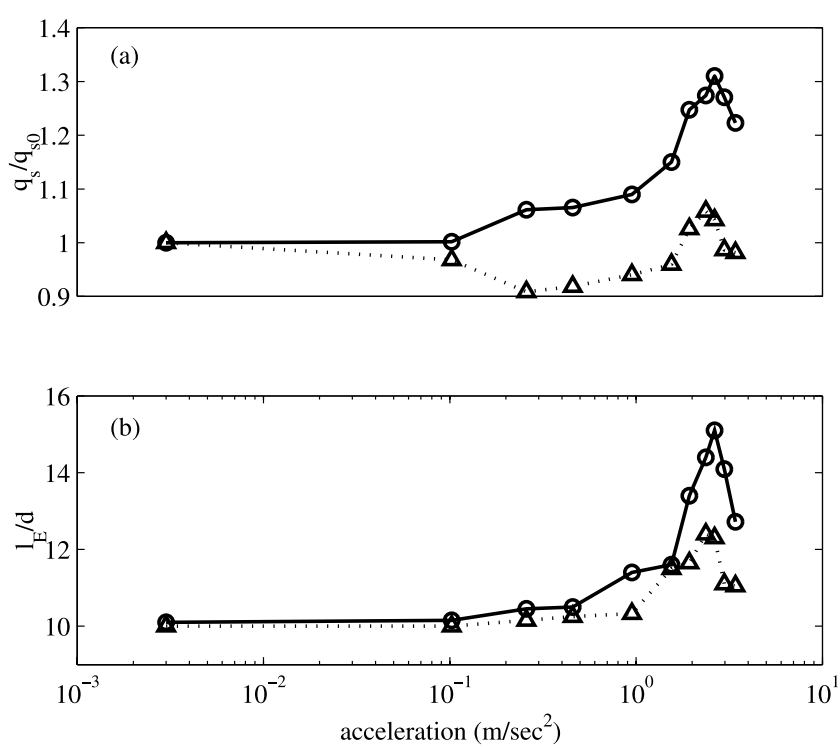

Figure 13. Sediment transport under prescribed step acceleration forcing. The step has a low free-stream velocity of $1.02 \mathrm{~m} / \mathrm{s}$ and high free-stream velocity of $2.18 \mathrm{~m} / \mathrm{s}$. (a) Sediment transport rate under various constant acceleration relative to that of their steady state condition and (b) corresponding nondimensional erosion depth, evaluated at free-stream velocity of $2.18 \mathrm{~m} / \mathrm{s}$ (circles) and free-stream velocity of $1.5 \mathrm{~m} / \mathrm{s}$ (triangles). 
the mobilized sediment to respond to the changing forcing conditions. Hence the sediment transport rate under steady state (i.e., fully saturated) is larger than at the same free stream velocity under a constant acceleration, where a finite duration for the development of transport is imposed, and where the pressure gradient is not sufficient to cause bed failure. When acceleration becomes greater than about $2.0 \mathrm{~m} / \mathrm{s}^{2}$, the effect of bed failure dominates and the corresponding sediment transport increases.

[43] Notice that the erosion depth and the corresponding sediment transport rate begin to decrease as the acceleration becomes greater than about $3.0 \mathrm{~m} / \mathrm{s}^{2}$. Because an acceleration of $3.0 \mathrm{~m} / \mathrm{s}^{2}$ for these cases corresponds to a duration of acceleration of only $\Delta t=0.39 \mathrm{~s}$, the observed decrease of the erosion depth and sediment transport rate again suggests a minimum development timescale of failure for the sediment bed in response to the changing flow.

[44] Using the step forcing, we have demonstrated that the bed failure is closely related to flow acceleration/ horizontal pressure gradient. However, it appears to require some time for both sediment transport and turbulent flow to adjust to the changing forcing conditions, and it also requires some shorter amount of time for the bed to completely fail or yield. We will refer to these two timescales as the saturation timescale and yield timescale, respectively. Because large acceleration often suggests a shorter duration of forcing, flow acceleration does not always enhance sediment transport. We believe that for small accelerations where the bed failure is not significant, the acceleration may cause a decrease in the sediment transport rate if the duration of forcing does not exceed the saturation timescale. On the contrary, when acceleration is large enough to approach or exceed the critical acceleration for bed failure, enhancing acceleration results in more severe failure and larger sediment transport. However, even for large accelerations, if the duration of forcing is shorter than the yield timescale, enhancing acceleration may again result in smaller sediment transport rate. Finally, we believe that the saturation and yield timescales are also functions of the magnitude of the flow velocity, the flow acceleration, and the fluid and sediment properties. Further systematic analysis on these issues and critical experiments are clearly motivated.

[45] One of the key features of unsteady sheet flows is that the amount of mobilized sediment and the location of the bed change with time. Bagnold [1956] argues that for steady flows the immersed weight of the sheet flow layer is related to the bed shear stress through a dynamic Coulomb yield criterion. If for some reason the bed shear stress increases slowly, then in the quasi-steady Bagnold paradigm, successive layers of stationary grains would be entrained into the flow until the newly increased immersed weight balanced the increase in the bed shear stress. However, results presented earlier also suggest that under very high accelerations the bed undergoes a sudden failure, analogous to the episodic avalanching of sand that is piled steeper than the critical angle of repose.

[46] Madsen [1974] performs an analysis on the momentary internal failure of sand bed under waves. He demonstrates that momentary bed failure under waves is more likely to be initiated by shear failure due to significant horizontal pressure gradient and less likely to be initiated by the vertical flow in the porous bed. Moreover, Madsen introduces a simple formula, based on the properties of the sand bed, to calculate the critical horizontal pressure gradient for momentary shear failure. On the basis of the two-phase equations, the horizontal momentum equation for the sediment-fluid mixture can be obtained by combining the horizontal momentum equation of the fluid and sediment phase. At the stationary bed, because all the particle motion vanishes and the fluid horizontal velocity can be also neglected for simplicity, a simplified mixture equation, similar to that used by Madsen [1974], becomes

$$
-\rho^{f} \frac{\partial u_{0}(t)}{\partial t}=\frac{\partial\left(\tau_{x z}^{f}+\tau_{x z}^{s}\right)}{\partial z}, \quad \text { at } z_{b}=-l_{E}
$$

Note that the horizontal pressure gradient term has been replaced by the free-stream acceleration. Equation (21) indicates that the gradient of total shear stress at bed must respond to the given flow acceleration, a surrogate for horizontal pressure gradient.

[47] To calculated the right-hand side of equation (21), Madsen [1974] further adopts the quasi-steady Bagnold paradigm [Bagnold, 1956], in which the gradient of total shear stress at bed is related to the normal stress through Coulomb failure and calculated by the immersed weight of sediment in fluid,

$$
\frac{\partial\left(\tau_{x z}^{f}+\tau_{x z}^{s}\right)}{\partial z}=\left(\rho^{s}-\rho^{f}\right) \bar{c}|g| \tan \phi, \text { at } z_{b}=-l_{E}
$$

Combining equations (21) and (22), and applying typical values for sediment density, concentration, and internal friction angle, this analysis suggests that an acceleration of approximately $0.4|g|$ is enough to cause catastrophic failure within the bed. Strictly speaking, because the closure presented in equation (22) is only valid in the fully developed steady state condition, the quasi-steady approach adopted by Madsen [1974] may be too simple to evaluate bed failure under highly unsteady conditions. The constitutive relations and closures used in the present two-phase model embody substantially more physics than Bagnold's descriptions. On the basis of numerical experiments of various step forcing using the sheet flow model (Figure 13), we also demonstrate that when the flow acceleration/ horizontal pressure gradient exceeds a critical value, rapid internal bed failure occurs.

[48] Sleath [1999] presents another analysis of the condition for the so-called "plug" formation motivated by his measured laboratory data [Dick and Sleath, 1991; Zala Flores and Sleath, 1998]. We note here that the "plug " that was described by Sleath [1999] is essentially the same as the bed failure described by Madsen [1974] and the present paper. On the basis of a somewhat more complicated quasi-steady analysis than Madsen [1974], Sleath [1999] introduces another parameter $S=U_{0} \omega /(s-1) g$ for the plug formation with $\omega$ the radial frequency. On the basis of his analysis and the measured data using acrylic particles $(d=$ $0.7 \mathrm{~mm}, s=1.14$ ), he suggests that the plug may form when $S$ is greater than a critical value (about 0.37 to 0.76 ). Since $U_{0} \omega$ defined in $S$ is equivalent to an averaged acceleration under sinusoidal wave forcing, a critical acceleration similar 

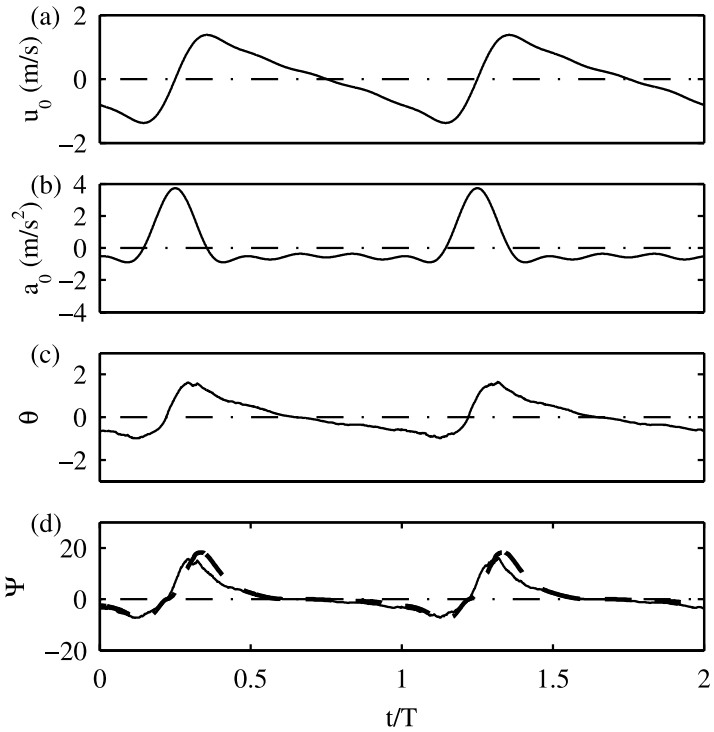

Figure 14. Sediment transport under saw-tooth wave of wave period $T=6.0 \mathrm{~s}$. (a) Prescribed free-stream velocity, (b) free-stream acceleration, and (c) nondimensional bed shear stress. (d) Nondimensional sediment transport rate using calculated nondimensional bed shear stress and Meyer-Peter Müller formula (solid curve) and two-phase sheet flow model (dashed curve).

to that of Madsen [1974] and the present paper is also reported by Sleath [1999].

[49] All these models indicate the existence of critical acceleration/horizontal pressure gradient for bed failure, although our simulation results suggest failure occurs in the dynamic system at a somewhat lower threshold acceleration than that suggested by the quasi-static analysis. Notice that Sleath [1999] also observes that the critical value for $S$ also depends on the degree of compaction of the sediment. On the basis of our numerical experiments, our model also indicates that the bed failure or the erosion depth under unsteady forcing depends on the compaction of the bed.

[50] In addition to laboratory experiments, recent field observations of Foster et al. [2002] also suggested the occurrence of plug flow during flow reversal. Because the bed failure can be a direct consequence of the large flow acceleration/horizontal pressure gradient, further measurements toward a better understanding of bed failure are necessary [e.g., Cox et al. 1991].

\section{Toward Parameterization}

[51] On the basis of the two-phase model, we demonstrate that whether the net sediment transport rate can be plausibly parameterized by the flow velocity depends on the wave shape. In particular, it is insufficient to simply use the instantaneous flow velocity to calculate the corresponding time-dependent sediment transport rate in a quasi-steady sense. This results from several mechanisms, such as the closures of fluid and sediment stresses, the effect of pressure gradient on the particles, and bed failure, that are not incorporated in the quasi-steady parameterization procedures. Hence our next objective is to explore a new calculation procedure for the sediment transport rate that incorporates essential unsteady effects.

[52] The nondimensional bed shear stress, usually called the Shields parameter,

$$
\Theta=\frac{\tau_{b}}{\left(\rho^{s}-\rho^{f}\right) g d}
$$

is perhaps the most well-accepted parameter in characterizing sediment transport processes [e.g., Ribberink, 1998]. We point out here that the location of the bed shear stress is clearly defined for fixed beds, but that for unsteady sheet flows the vertical location of the stationary bed changes in time. In addition, the fluid and granular phase shear stresses vary with vertical position and time. Since the closures of both the fluid and particle stresses have been incorporated in the model, the total shear stress at any level within the sheet, including the bed shear stress, becomes part of the solution of the model. Here the term "bed shear stress" is used to indicate the instantaneous total shear stress at the uppermost level of the stationary bed. Figure $14 \mathrm{c}$ shows the calculated time history of the nondimensional bed shear stress, which we shall refer to as $\theta(t)$, the generalized Shields parameter, due to the saw-tooth forcing considered earlier. Notice that unlike the time history of free-stream velocity shown in Figure $14 \mathrm{a}$, the time history of $\theta(t)$ is not symmetric with respect to the positive and negative phase. Therefore a formula that calculates $\theta(t)$ solely based on the free-stream velocity is inappropriate. Moreover, since the shape of $\theta(t)$ in Figure $14 \mathrm{c}$ is similar to the time history of the sediment transport rate presented in Figure 14c, we are motivated to estimate the sediment transport rate by substituting the generalized Shields parameter, $\theta(t)$, into the Meyer-Peter Müller formula,

$$
\Psi(t)=8[\theta(t)-0.05]^{3 / 2}
$$

The resulting nondimensional sediment transport rate (solid curve in Figure 14d) closely resembles the direct calculations from the sheet flow model (dashed curve). Therefore the present sheet flow model indicates that it is, at least qualitatively, reasonable to apply a steady state formula in a quasi-steady manner to an unsteady condition as long as the corresponding bed shear stress can be accurately estimated.

[53] Owing to improved computational power, phaseresolving models for surfzone hydrodynamics and sediment transport have become popular in recent years [e.g., Wei and Kirby, 1995; Lin and Liu, 1998]. Therefore it would be extremely useful to develop a reliable and efficient calculation procedure for instantaneous sediment transport rate that incorporates essential unsteady effects. On the basis of the two-phase model, we believe that given the instantaneous bed shear stress, a quasi-steady approach may be applied to estimate the instantaneous sediment transport rate. However, such calculation procedure must rely on accurate prediction on instantaneous bed shear stress which may not be parameterized solely by the flow velocity quasisteadily.

[54] For a predictive nearshore morphological model, a more efficient approach to calculate the bed shear stress 
than the present two-phase model is necessary. Nielsen [1992] and Nielsen and Callaghan [2003] propose a formula to calculate the instantaneous bed shear stress that explicitly incorporates flow velocity and acceleration. However, the development of a more physical-based, yet efficient calculation procedure for instantaneous bed shear stress is nontrivial [e.g., Grant and Madsen, 1979; Trowbridge and Madsen, 1984]. Evidently, in a wave boundary layer flow, the near-bed flow velocity would be completely in phase with the free-stream velocity if the flow were inviscid. In other words, an accurate prediction of bed shear stress must rely on reasonable closure on fluid and sediment stresses. For this, whether we can cost-effectively parameterize (or simplify) the vertical distribution of the fluid turbulence and particle intergranular interactions across the wave boundary layer becomes crucial and requires future work.

\section{Conclusion}

[55] Using a recently developed two-phase model, we examine in detail the effects of wave shape on sediment transport. We find that for certain wave shapes, time-dependent sediment transport cannot be completely parameterized by the instantaneous free-stream velocity. Examinations of the sheet flow response to flow forcing typical of asymmetric and skewed waves indicate a net sediment transport in the direction of wave propagation.

[56] Using a closure of particle intergranular stress based on the kinetic theory of granular flow in the two-phase equations, we examine the role of the particle collisional stress in the sediment momentum budget. The particle collisional stress tends to effectively balance the drag force in the sediment horizontal budget and thus enhance the relative effect of horizontal pressure gradient on the sediment dynamics. Moreover, numerical experiments indicate that catastrophic internal bed failure is a direct consequence of large horizontal pressure gradient (or large flow acceleration).

[57] Using the sheet flow model, we demonstrate that a power law, which can be as simple as the MeyerPeter Müller formula, can predict the sediment transport reasonably well as long as accurate description for the corresponding instantaneous bed shear stress can be obtained. We believe these results will provide new guidelines toward an improved parameterization for sediment transport rate under unsteady conditions.

[58] Finally, we note here that all the simulations presented in this work were for coarse sand with a diameter of $1.1 \mathrm{~mm}$. For such coarse sediment, collisions between grains are dominated by the grains' inertia, the fluid plays a minor role, and the kinetic theory for granular flow appropriately captures the dominant momentum and energy pathways. More typical beach sand, of diameter $0.2 \mathrm{~mm}$ for example, may behave differently due to the relatively greater influence of the interstitial fluid upon the grain-tograin interactions. We believe one of the most important extensions of this and previous work would be to model sediment transport of fine and medium sands using the twophase approach with the closure of particle stresses based on particle fluctuation energy and concentrated viscous suspension models.
[59] Acknowledgments. We gratefully acknowledge the financial supports of the National Ocean Partnership Program and the Department of Civil and Environmental Engineering, University of Delaware. Numerous discussions with James Jenkins and Philip Liu during the development of the sheet flow model are appreciated. We also wish to thank James Kirby, John Warner, Jon Warrick, and Fernanda Hoefel for their useful comments on earlier versions of this manuscript.

\section{References}

Asano, T. (1995), Sediment transport under sheet-flow conditions, J. Waterw. Port Coast. Ocean Eng., 121(5), 1-8.

Bagnold, R. A. (1956), The flow of cohesionless grains in fluid, Philos. Trans. R. Soc. London, Ser. A, 249(964), 235-297.

Bailard, J. (1981), An energetic total load sediment transport model for a plane sloping beach, J. Geophys. Res., 86, 10,938-10,954

Bocquet, L., W. Losert, D. Schalk, T. C. Lubensky, and J. P. Gollub (2002), Granular shear flow dynamics and forces: Experiment and continuum theory, Phys. Rev. E, 65(1), 011307.

Butt, T., and P. Russell (1999), Suspended sediment transport mechanism in high-energy swash, Mar. Geol., 161, 361-375.

Cox, D. T., N. Kobayashi, and M. Hajime (1991), Effect of fluid acceleration on sediment transport in surf zone, Coastal Sed., 91, 447-461.

Dick, J. E., and J. F. A. Sleath (1991), Velocities and concentrations in oscillatory flow over beds of sediment, J. Fluid Mech., 233, 165-196.

Doering, J. C., B. Elfrink, D. M. Hanes, and G. Ruessink (2000), Parameterization of velocity skewness under waves and its effect on cross-shore sediment transport, paper presented at 27th Coastal Engineering Conference, Am. Soc. of Civ. Eng., Reston, Va.

Dohmen-Janssen, C. M., and D. M. Hanes (2002), Sheet flow dynamics under monochromatic nonbreaking waves, J. Geophys. Res., 107(C10), 3149, doi:1029/2001JC001045

Drake, T. G., and J. Calantoni (2001), Discrete particle model for sheet flow sediment transport in the nearshore, J. Geophys. Res., 106(C9), 19,85919,868 .

Drew, D. A. (1976), Production and dissipation of energy in the turbulent flow of a particle-fluid mixture, with some results on drag reduction, J. Appl. Mech., 43, 543-547.

Elgar, S., and R. T. Guza (1985), Observations of bispectra of shoaling surface gravity waves, J. Fluid Mech., 161, 425-448.

Elgar, S., E. L. Gallagher, and R. T. Guza (2001), Nearshore sandbar migration, J, Geophy. Res., 106(C6), 11,623-11,627.

Elghobashi, S. E., and T. W. Abou-Arab (1983), A two-equation turbulence model for two-phase flows, Phys. Fluids, 26(4), 931-938.

Foster, D. L., R. A. Holman, and A. J. Bowen (2002), Field evidence for plug flow, Eos Trans. $A G U, 83(47)$, Fall Meet. Suppl., Abstract OS72C-02.

Gallagher, E. L., S. Elgar, and R. T. Guza (1998), Observations of sand bar evolution on a natural beach, J. Geophys. Res., 103(C2), 3203-3215.

Grant, W. D., and O. S. Madsen (1979), Combined wave and current interaction with a rough bottom, J. Geophys. Res., 84(C4), 1797-1808.

Hanes, D. M., and D. A. Huntley (1986), Continuous measurements of suspended sand concentration in a wave dominated nearshore environment, Cont. Shelf Res., 6, 585-596.

Hanes, D. M., and D. L. Inman (1985a), Observations of rapidly flowing granular-fluid materials, J. Fluid Mech., 150, 357-380.

Hanes, D. M., and D. L. Inman (1985b), Experimental evaluation of a dynamic yield criterion for granular-fluid flow, J. Geophys. Res., 90(B5), 3670-3674.

Hoefel, F., and S. Elgar (2003), Wave-induced sediment transport and sandbar migration, Science, 299, 1885-1887.

Hsu, T.-J., J. T. Jenkins, and P. L.-F. Liu (2003), On two-phase sediment transport: Dilute flow,J. Geophys. Res., 108(C3), 3057, doi:10.1029/2001JC001276 . Hsu, T.-J., J. T. Jenkins, and P. L.-F. Liu (2004), On two-phase sediment transport: Sheet flow of massive particles, Proc. R. Soc. London, Ser. A, 460(2048), doi:10.1098/rspa.2003.1273

Jenkins, J. T., and D. M. Hanes (1998), Collisional sheet flows of sediment driven by turbulent fluid, J. Fluid Mech., 370, 29-52.

Jenkins, J. T., and S. B. Savage (1983), A theory for the rapid flow of identical, smooth, nearly elastic particles, J. Fluid Mech., 370, 29-52.

Jenkins, J. T., P. A. Cundall, and I. Ishibashi (1989), Micromechanics modeling of granular material, in Powders and Grains, edited by J. Biarez and R. Gourves, pp. 257-264, A. A. Balkema, Brookfield, Vt.

King, D. B. (1990), Studies in oscillatory flow bed load sediment transport, Ph.D. thesis, Univ. of Calif., San Diego.

Lin, P., and P. L.-F. Liu (1998), A numerical study of breaking waves in the surf zone, J. Fluid Mech., 359, 239-264.

Madsen, O. S. (1974), Stability of a sand bed under breaking waves, paper presented at 14th Coastal Engineering Conference, Am. Soc. of Civ. Eng., Reston, Va. 
Masselink, G., and M. G. Hughes (1998), Field investigation of sediment transport in the swash zone, Cont. Shelf Res., 18, 1179-1199.

Nielsen, P. (1992), Coastal Bottom Boundary Layers and Sediment Transport, World Sci., River Edge, N. J.

Nielsen, P. (2002), Shear stress and sediment transport calculations for swash zone modeling, Coastal Eng., 45, 53-60.

Nielsen, P., and D. P. Callaghan (2003), Shear stress and sediment transport calculations for sheet flow under waves, Coastal Eng., 47, 347-354.

Puleo, J. A., K. T. Holland, N. G. Plant, D. N. Slinn, and D. M. Hanes (2003), Fluid acceleration effects on suspended sediment transport in the swash zone, J. Geophys. Res., 108(C11), 3350, doi:10.1029/ 2003JC001943.

Ribberink, J. S. (1998), Bed-load transport for steady flows and unsteady oscillatory flows, Coastal Eng., 34, 59-82.

Richardson, J. F., and W. N. Zaki (1954), Sedimentation and fluidization: 1, Trans. Inst. Chem. Eng., 32, 35-53.

Sleath, J. F. A. (1999), Conditions for plug formation in oscillatory flow, Cont. Shelf Res., 19, 1643-1664.

Sumer, B. M., A. Kozakiewicz, J. Fredsøe, and R. Deigaard (1996), Velocity and concentration profiles in sheet-flow layer of movable bed, J. Hydrol. Eng., 122(10), 549-558.
Torquato, S. (1995), Nearest-neighbor statistics for packings of hard spheres and disks, Phys. Rev. E, 51, 3170-3182.

Trowbridge, J., and O. S. Madsen (1984), Turbulent wave boundary layers: 2. Second-order theory and mass transport, J. Geophys. Res., 89(C5), $7999-8007$.

Wei, G., and J. T. Kirby (1995), A time-dependent numerical code for extended Boussinesq equations, J. Waterw. Port Coastal Ocean Eng., $121,251-261$.

Young, J., and A. Leeming (1997), A theory of particle deposition in turbulent pipe flow, J. Fluid Mech., 340, 129-159.

Zala Flores, N., and J. F. A. Sleath (1998), Mobile layer in oscillatory sheet flow, J. Geophys. Res., 103(C6), 12,783-12,793.

D. M. Hanes, Western Coastal and Marine Geology, U.S. Geological Survey Pacific Science Center, 400 Natural Bridges Drive, Santa Cruz, CA 95060, USA. (hanes@ufl.edu)

T.-J. Hsu, Woods Hole Oceanographic Institution, Applied Ocean Physics and Engineering Department, MS 11, Woods Hole, MA 02543, USA. (thsu@whoi.edu) 